\title{
Computational Retinal Imaging via Binocular Coupling and Indirect Illumination
}

\author{
Everett Lawson Jason Boggess Siddharth Khullar Alex Olwal Gordon Wetzstein Ramesh Raskar \\ MIT Media Lab
}
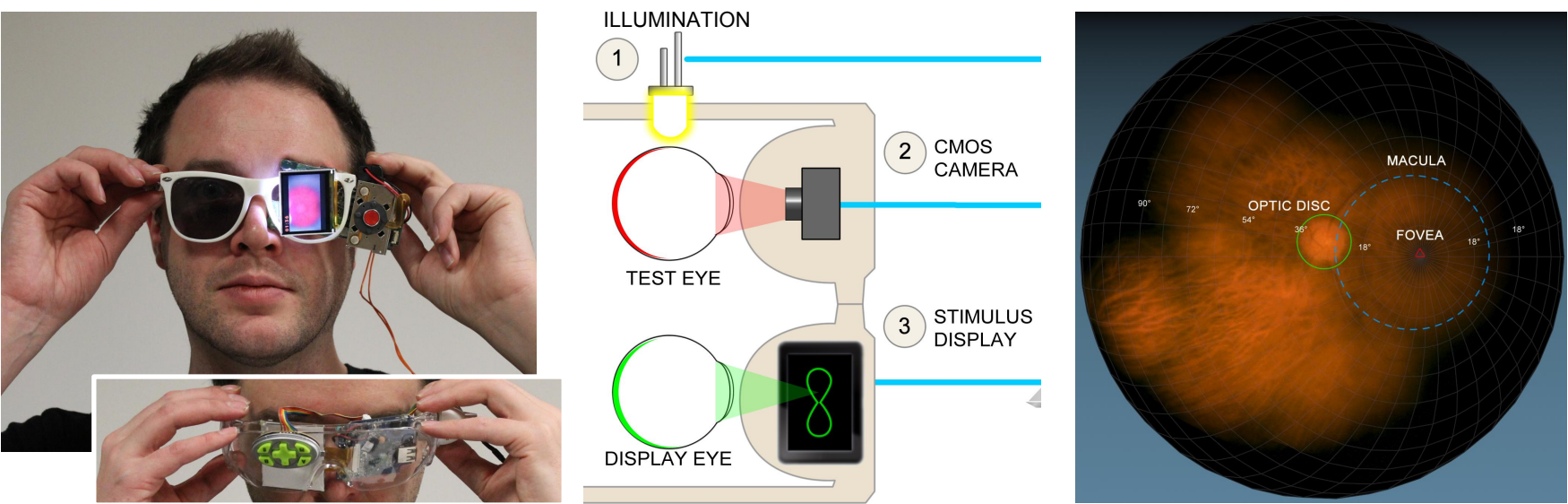

Figure 1: How can we capture images of the human retina using a standalone, hand-held and user-interactive device? Our system comprises a unique co-design of a low-cost CMOS camera, programmable stimulus control, and indirect diffusive illumination to create an interactive and portable alternative to conventional retinal (fundus) imaging devices. Using this imaging platform, we add the dimension of userinteraction to the field of wireless health and tele-medicine by enabling unsupervised and efficient retinal imaging for measuring longitudinally modulating parameters within the human retina.

Introduction and Overview The retina is a complex lightsensitive tissue that is an essential part of the human visual system. It is unique, as it can be optically observable with non-invasive methods through the eye's transparent elements. This has inspired a long history of retinal imaging devices for examination of optical function [Van Trigt 1852; Yates 2011] and for diagnosis of many of the diseases that manifest in the retinal tissue, such as diabetic retinophathy, hypertension, HIV/AIDS related retinitis, and age-related macular degeneration. These conditions are some of leading causes of blindness, especially in the developing world, but can often be prevented if screened and diagnosed in early stages.

Unfortunately, the majority of retinal imaging devices employ high quality optical elements for illumination and observation and require precise alignment with the eye [Keeler 1997; Abràmoff et al. 2010]. Available devices are bulky, expensive, and virtually not accessible in developing countries. We present an inexpensive, selfdirected, interactive device to capture and visualize images of the retina (Figure 1). For this purpose, we exploit a combination of two novel ideas to overcome the alignment and illumination requirements of traditional devices. First, we use indirect diffuse illumination in a small form factor close to the eye. This avoids the challenges of direct pupillary illumination via focused beams. Second, we put the user in the loop and exploit the natural biological coupling of binocular vision. We use a close-up display on one eye to help the user self-align the pupil. In addition, we lock the gaze and focus of the same eye for convenient imaging of the other eye.

Approach Our prototypes use ultrabright $1 W$ dichromatic white LEDs with a luminous efficacy of $120 \mathrm{~lm} / W$ mounted near the user's temple, to indirectly illuminate the retina through the tissue. The image of the illuminated retina is projected out through the eye, where it is captured by a camera for a real-time live video feed. In our standalone prototypes, which fit in a pair of modified glasses, we provide the user, or an observer, with a live view of the retina. To capture wide field-of-view retinal panoramas, our other prototypes use a tethered camera and software for real-time processing and image stitching. Our software exploits binocular coupling to gaze-lock the eyes, such that the rotation of the test eye can be computationally controlled through stimulus patterns shown to the display eye (see Fig. 1).

Potential Impact and Discussion Many of the leading diseases, on a global scale, of both the eye and the body manifest on and within the substructures of the retinal lining. As an optical system, the human eye allows for direct screening using non-invasive imaging technology. Unfortunately, todays retinal imaging devices are bulky, expensive, and require highly trained ophthalmologists and specialized capture conditions. Our system is compact, low-cost, requires no moving parts, and utilizes a novel combination of indirect diffuse illumination through the side of the eye coupled with, a camera, and a computationally controlled display, which requires no special training, can be a self-examination, and enables retinal imaging into new form of graphic renderings.

With our work, we demonstrate the ability to use computational imaging and interactive techniques for low-cost retinal imaging via binocular coupling and indirect illumination. Although these are only the first steps toward a robust diagnostic tool, the positive feedback we have received from ophthamalogists indicates great promise and many future opportunities to map graphics research to this important health task. We hope our work will inspire further graphics and user interaction research for modeling human anatomy with low-cost devices that will eventually impact global health in remote parts of the world.

\section{References}

ABRÀmofF, M., GARVIN, M., AND SONKA, M. 2010. Retinal imaging and image analysis. Biomedical Engineering, IEEE Reviews in 3, 169-208.

KEELER, C. 1997. 150 years since babbage's ophthalmoscope. Archives of ophthalmology 115, 11, 1456.

VAN TRIGT, A. 1852. De oogspiegel. Nederlandisch Lancet, third series, Utrecht $1853,417-509$.

Yates, Paul Andrew; Tran, K., 2011. Hand-held portable fundus camera for screening photography, March. 